\title{
Studies on the reversible enzyme reaction of rabbit muscle glycogen phosphorylase $b$ using isothermal titration calorimetry
}

Kármen Szabó, Lili Kandra, Gyöngyi Gyémánt*

Department of Inorganic and Analytical Chemistry, University of Debrecen, Debrecen, Hungary

*Corresponding author: Gyöngyi Gyémánt

e-mail: gyemant@science.unideb.hu

Address: Department of Inorganic and Analytical Chemistry, University of Debrecen, H-4002

Debrecen, POBox 400

Telephone: +3652512900

Fax number : +3652518660

ORCID: Gyöngyi Gyémánt: 0000-0003-2374-3408

Declarations of interest: none 


\begin{abstract}
Glycogen phosphorylase enzymes (GP) catalyse reversible reactions; the glucose transfer from glycogen to inorganic phosphate $\left(\mathrm{P}_{\mathrm{i}}\right.$, phosphorolysis) or the reverse glucose transfer from glucose-1-phosphate (G-1-P) to glycogen (synthesis). Rabbit muscle GPb (rmGPb) was used as a model enzyme to study the reversible enzyme reaction. To follow both directions of this reversible reaction, we have developed a novel isothermal titration calorimetry (ITC) method for the determination of the direct reaction rate. The preference of forward or reverse reaction was ensured by the 0.1 or 10 concentration ratios of $\mathrm{G}-1-\mathrm{P} / \mathrm{P}_{\mathrm{i}}$, respectively. Substrate specificity was studied using different maltooligosaccharides and glycogen. Based on the $K_{M}$ values, glycogen and 2-chloro-4-nitrophenyl maltoheptaoside (CNP-G7) were found to be analogous substrates, which allowed to optimize the method by taking advantage of the CNP chromophore being detectable in HPLC. In case of CNP-G7, substrate inhibition was observed and characterised by $K_{i}$ of $23 \pm 7 \mathrm{mM}$. Inhibition of human GP is a promising strategy for the treatment of diabetes. Our ITC measurements have confirmed that caffeine and glucopyranosylidene-spirothiohydantoin (GTH), as known GPb inhibitors, inhibit the rmGPb-catalysed reversible reaction in both directions. $K_{i}$ values obtained in the direction of synthesis $(1.92 \pm 0.14 \mathrm{mM}$ for caffeine and $11.5 \pm 2.0 \mu \mathrm{M}$ for $\mathrm{GTH}$ ) have been shown to be in good agreement with the $K_{i}$ values obtained in the direction of phosphorolysis $(4.05 \pm 0.26 \mathrm{mM}$ for caffeine and $13.8 \pm 1.6 \mu \mathrm{M}$ for GTH). The higher difference between the inhibition constants of caffeine was explained by the non-competitive mechanism. The described ITC method using the developed experimental design and reaction conditions is suitable for activity measurements of different phosphorylase enzymes on various substrates and is applicable for inhibition studies as well.
\end{abstract}

Keywords: glycogen phosphorylase; isothermal titration calorimetry; reversible enzyme reaction; substrate inhibition; activity measurement. 


\section{Introduction}

Glycogen is the major storage form of carbohydrates in animals. It provides glucose units for glycolysis within the muscle, while liver glycogen maintains the blood glucose level. Glycogen phosphorylase (GP), the principal enzyme of glycogen metabolism, is a possible target for the treatment of diabetes. Muscle GP enzyme exists in phosphorylated, active (GPa) and dephoshorylated, inactive $(\mathrm{GPb})$ form, which is active only in the presence of its allosteric activators including adenosine monophosphate (AMP). Since GP catalyses a reversible reaction, different activity measurement methods have been developed for the forward or reverse reaction. An early and complicated method was applied by Tanabe for following the phosphorolytic reaction [1]. In this case, the phosphate content of G-1-P was measured after a hydrolysis step. In the direction of phosphorolysis, glycogen and phosphate substrates are often used in a coupled enzyme assay (with phosphoglucomutase and glucose-6-phosphate dehydrogenase). The reduced coenzyme (NADH or NADPH) generated in the redox reaction is determined by spectrophotometry [2]. This system is less convenient for inhibition studies due to the presence of the two additional enzymes, which can also bind to the inhibitor. Recently an ESI-MS method has been suggested for the quantification of G-1-P produced during the degradation of the glycogen or maltooligomer substrates to promote the proteomic study of sugar phosphorylases [3]. There is an additional option for the measurement of G-1-P released from glycogen, namely the use of an anion-exchange HPLC method on Carbopack PA10 column with pulsed amperometric detection [4]. Fluorescent substrates (pyridylaminated maltohexaose and some of its derivatives) and HPLC measurement on polymer based HILIC column have recently been utilized for probing the catalytic site of rabbit muscle $\mathrm{GPb}(\mathrm{rmGPb})$ [5].

Several research groups study the enzyme in the direction of glycogen synthesis which doesn't correspond with the physiological reaction. The most frequently applied method is based on the colorimetric determination of inorganic phosphate deriving from G-1-P during the glycogen synthesis. This colour reaction is very sensitive, but the intensity of the colour is influenced by several parameters, which can deteriorate the accuracy [6]. Radioactive measurements of the product obtained from the synthesis reaction catalysed by GP require special equipment and radioactive labelled G-1-P substrate [7]. In 2009 Makino et al. reported a method in which glycogen phosphorylase activity was determined by HPLC in the direction of glycogen synthesis. 
This assay was carried out in the presence of glucose-1-phosphate and maltooligosaccharides as substrates which had been labelled with chromophore group in a pyridylamination reaction [8].

Our aim was to develop a method suitable for activity measurement in both directions of the enzyme reaction. Isothermal titration calorimeters can be considered as universal detectors because of the change in heat occurring in almost every chemical reaction. There are further advantages of isothermal titration calorimetry (ITC): natural substrates can be utilized without derivatization and the applied measurement method is not sensitive to the composition of reaction mixture. Rabbit muscle glycogen phosphorylase b (EC 2.4.1.1) was chosen as a model enzyme since most of the inhibition measurements of GP have been carried out with rmGP enzyme (see BRENDA database). Considering that GPb is inactive, experiments were accomplished in the presence of AMP [9].

In this work we studied the effect of two known GP inhibitors (caffeine as allosteric and glucopyranosylidene-spiro-thiohydantoin (GTH) as competitive inhibitor) in both directions of the catalysed reaction using the same ITC method for the measurement of the reaction rate.

\section{Results and discussion}

\subsection{Characterization of synthetic and phosphorolytic reactions of $\mathrm{rmGPb}$ by ITC}

Reaction rate measurements were performed by ITC in both directions of the rmGPb-catalysed reversible reaction. Synthesis is an exothermic process, while phosphorolysis is an endothermic one resulting in negative (Figure 1A) and positive (Figure 1B) heat flow, respectively.
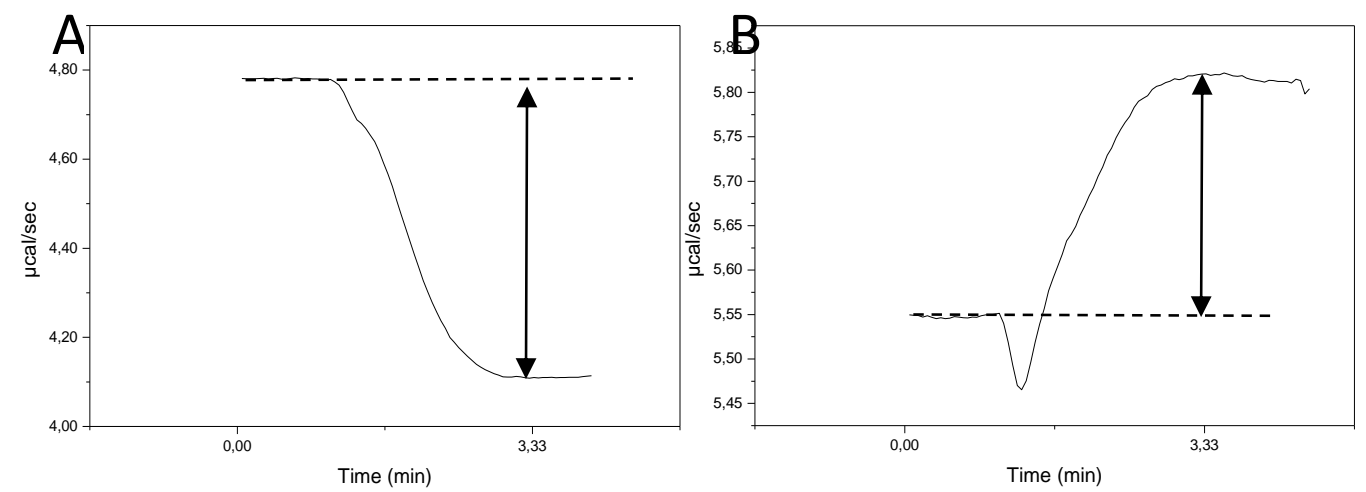

Figure 1. Representative ITC curves of a synthesis (A) and a phosphorolysis (B) reaction catalysed by rmGP enzyme. $0.5 \%$ glycogen was applied as substrate. Arrows symbolize the measured heat flow, $d Q / d t$, which is equal to the maximal deflection from the baseline. 
The reaction rate is proportional to the maximal heat flow $(d Q / d t)$ of the reaction and it is not relevant to the reaction rate whether the heat signal is positive or negative. Single injection ITC experiments were carried out where the enzyme solution (in the injector) was titrated into the sample cell containing the proper dilutions of the substrate solution.

Equation (1) allows the reaction rate to be calculated from the measured heat flow [10]:

$$
v=\frac{d[P]}{d t}=\frac{1}{V \times \Delta H} \times \frac{d Q}{d t}
$$

where $d[P] / d t$ is the rate of product formation, $V$ is the cell volume, $d Q / d t$ is the heat flow, and $\Delta H$ is the enthalpy change. The $(V \times \Delta H)^{-1}$ value is constant using the same system (cell and enzyme concentration, substrate) and temperature, hence $d Q / d t$ is a measure of the reaction rate. Initial rate $\left(v_{0}\right)$ was determined in our experiments since the maximal deflection was obtained at the beginning of the reaction (1-2 min after the injection) where the conversion of substrate is less than $10 \%$. Therefore, a single injection resulted in a single data pair of $v_{0}$ in the function of $[S]$.

\subsection{Selection of effective activator concentration}

The inactive free $\mathrm{GPb}$ enzyme may be activated by the addition of its well-known allosteric activator (AMP). In order to select the appropriate AMP concentration, the activation effect of AMP was studied on CNP-G7 substrate in the direction of phosphorolysis as well as of synthesis

under pseudo-first order conditions provided by the high concentration of the second substrate $\left(\mathrm{P}_{\mathrm{i}}\right.$ and G-1-P, respectively). We got $50 \mu \mathrm{M}$ and $26 \mu \mathrm{M}$ for half maximal effective concentration $\left(\mathrm{EC}_{50}\right)$ measured in the direction of phosphorolysis and synthesis, respectively. $E C_{50}$ values of literature for glycogen substrate fall in the range of $20-90 \mu \mathrm{M}$, which is in good accordance with our results $[11,12]$.

The experimental curve reached the saturation at $1 \mathrm{mM}$ concentration of AMP (data are not shown), which is in good agreement with the use of 1-2 mM AMP as activator in the experiments published earlier [1], [12-14]. Based on our results, 1mM AMP was applied in each experiment. 


\subsection{Study of substrate specificity}

Substrate specificity was studied in view of developing a new enzyme assay.

ITC measurements were carried out on maltooligomers (maltopentaose: G5, maltoheptaose: G7), glycogen and a chromophore containing maltoheptaoside substrate (CNP-G7) along with $\mathrm{P}_{\mathrm{i}}$ as a second substrate to ensure the phosphorolytic reaction. Figure 2. represents the experimental ITC curves obtained for G5 of different concentrations. The maximum values of the curves gave the heat flow, which is proportional to the reaction rate and were applied for the determination of $K_{M}$ and $v_{\max }$.

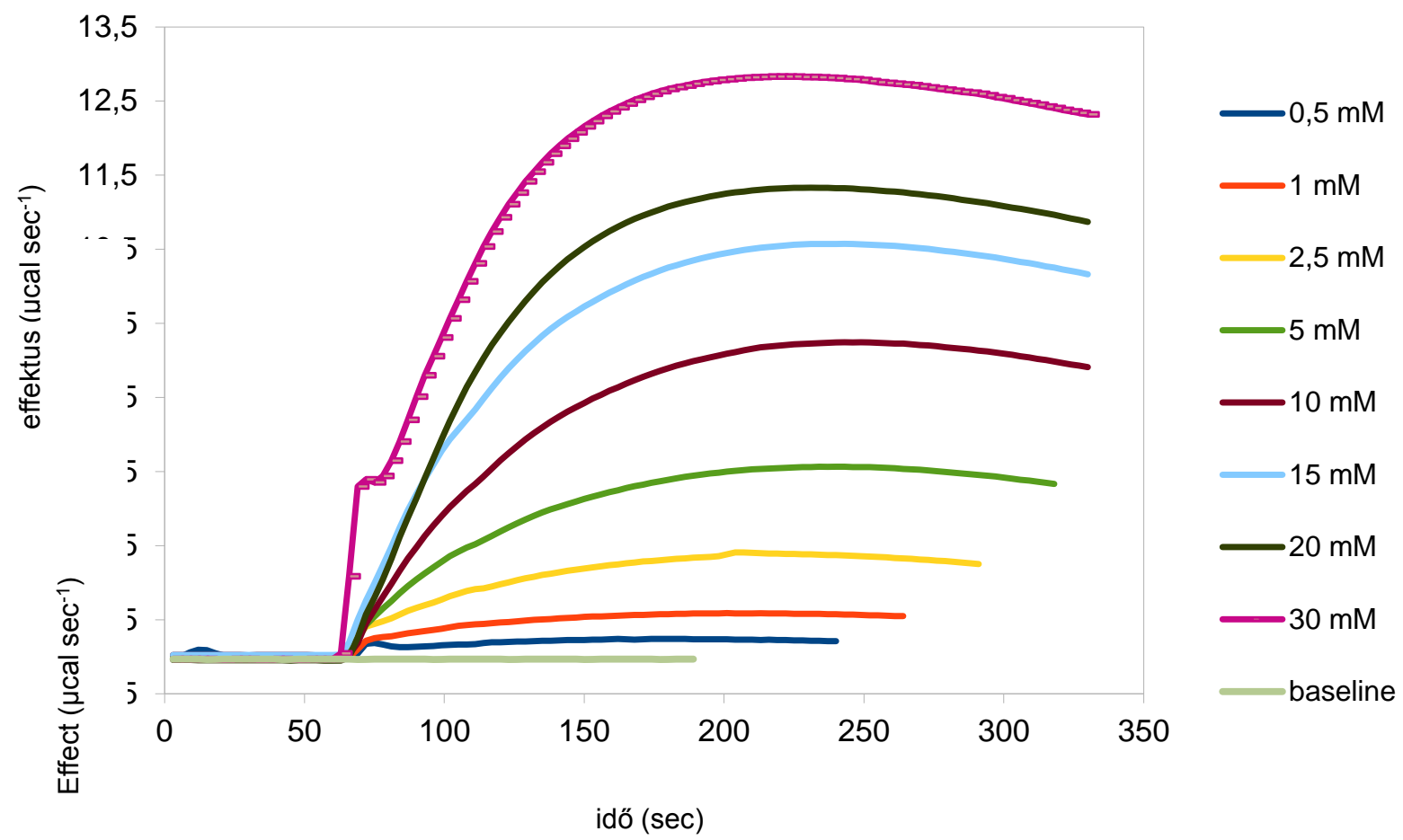

Figure 2. Experimental ITC thermograms for GPb-G5 reactions at different substrate concentrations. Measurements were carried out at $37^{\circ} \mathrm{C}$. Substrate concentration ranged from 0.5 to $30 \mathrm{mM}$ and $0.37 \mu \mathrm{M}$ enzyme was applied.

In order to compare the catalytic effectiveness of rmGPb for the four substrates being studied, $k_{c a t} / K_{M}$ ratios were determined. First, the maximal reaction rates $\left(v_{\max }\right)$ were calculated from our experimental heat flow data using Equation (1), where the total cell volume was $V=204 \mu \mathrm{L}$ and the $\Delta H$ value, obtained from a single injection measurement, was $2.32 \mathrm{kcal} / \mathrm{mol}$. G5 substrate was selected for the determination of $\Delta H$ since the pentamer includes only a single glycosidic bond 
cleavable by $\mathrm{GPb}$ enzyme. The subsequent step was to divide $v_{\max }$ by the initial enzyme concentration $[E]_{0}=0.37 * 10^{-6} \mathrm{M}$ (same in each experiment), which resulted in $k_{\text {cat }}$ values.

The results of our ITC measurements and data from literature are listed in Table 1.

Table 1. Kinetic constants of rmGPb enzyme on different substrates

\begin{tabular}{|c|c|c|c|c|c|c|}
\hline \multirow{3}{*}{ Substrate } & \multicolumn{3}{|c|}{ Measured by ITC } & \multicolumn{3}{|c|}{ Data of literature } \\
\hline & phosphoro & & synthesis & & phosphorolysis & synthesis \\
\hline & $\mathrm{K}_{\mathrm{M}}(\mathrm{mM})$ & $\begin{array}{l}\mathrm{kcat} / \mathrm{K}_{\mathrm{M}} \\
\mu \mathrm{calL}(\mathrm{sMg})^{-1}\end{array}$ & $\mathrm{~K}_{\mathrm{M}}(\mathrm{mM})$ & $\begin{array}{l}\mathrm{kcat} / \mathrm{K}_{\mathrm{M}} \\
\mu \mathrm{calL}(\mathrm{sMg})^{-1}\end{array}$ & $\mathrm{~K}_{\mathrm{M}}(\mathrm{mM})$ & $\mathrm{K}_{\mathrm{M}}(\mathrm{mM})$ \\
\hline G5 & $20.2 \pm 0.3$ & $2.3 * 10^{-4}$ & nd & nd & 9.1 & 38 \\
\hline G7 & $6.0 \pm 0.6$ & $3.0 * 10^{-4}$ & nd & nd & 7.1 & 31 \\
\hline CNP-G7 & $2.3 \pm 0.6^{b}$ & $6.6 * 10^{-4}$ & $15.2 \pm 2.1$ & $0.16 * 10^{-4}$ & nd & nd \\
\hline glycogen & $1.5 \pm 0.1^{\mathrm{c}}$ & nd & nd & nd & 0.15 & 0.6 \\
\hline
\end{tabular}

${ }^{\mathrm{a}}[1]$

${ }^{\mathrm{b}}[\mathrm{S}]_{0.5}$ because CNP-G7 reaction does not obey the Michaelis-Menten kinetics

${ }^{\mathrm{c}} \mathrm{mg} / \mathrm{ml}$

nd: no data

\pm values are the fitting error

Additional measurements were performed in the direction of synthesis in the presence of G-1-P and CNP-G7 due to the lack of kinetic data for chain lengthening reaction utilizing CNP-G7 as substrate. Kinetic parameters $K_{M}=15.2 \pm 2.2 \mathrm{mM}$ and $k_{c a t} / K_{M}=0.25^{*} 10^{3} \mathrm{~s}^{-1} \mathrm{M}^{-1}$ were determined for synthesis reaction. Substrate specificity proved to be very low in the synthesis reaction of CNPG7. The ratio of $K_{M}$ values obtained from the phosphorolysis and synthesis reactions of CNP-G7 is similar to the ratio published for maltooligomers. Comparing the kinetic parameters $\left(K_{M}\right.$ and $k_{c a t} / K_{M}$ ) obtained for G7 and CNP-G7, preferential effect of CNP was observed (see Table 1).

Even though rmGP is a well-studied enzyme, literature currently includes only a single article (published by Tanabe et al. [1]) containing $K_{M}$ data for maltooligomer substrates with different chain lengths. These values have been determined for both directions of the reversible reaction. Although the order of magnitude was similar, $K_{M}$ values obtained for the same substrate in this work differed from those reported by Tanabe, which can be explained by the different phosphate and AMP concentrations. Comparison of $K_{M}$ values derived from the two reactions revealed that lower substrate concentration is necessary for phosphorolysis than for synthesis to achieve the half maximal reaction rate. It is in accordance with the physiological function of mammalian 
glycogen phosphorylase which is the breakdown of glycogen. Catalytic effectiveness $\left(k_{c a t} / K_{M}\right)$ of linear substrates are similar in magnitude, while this value for glycogen is much higher due to the numerous nonreducing ends (Table 1).

\subsection{Substrate inhibition of $\mathrm{rmGPb}$}

During data evaluation substrate inhibition was observed in the phosphorolysis reaction of the chromophore containing substrate, CNP-G7 (Fig. 3).

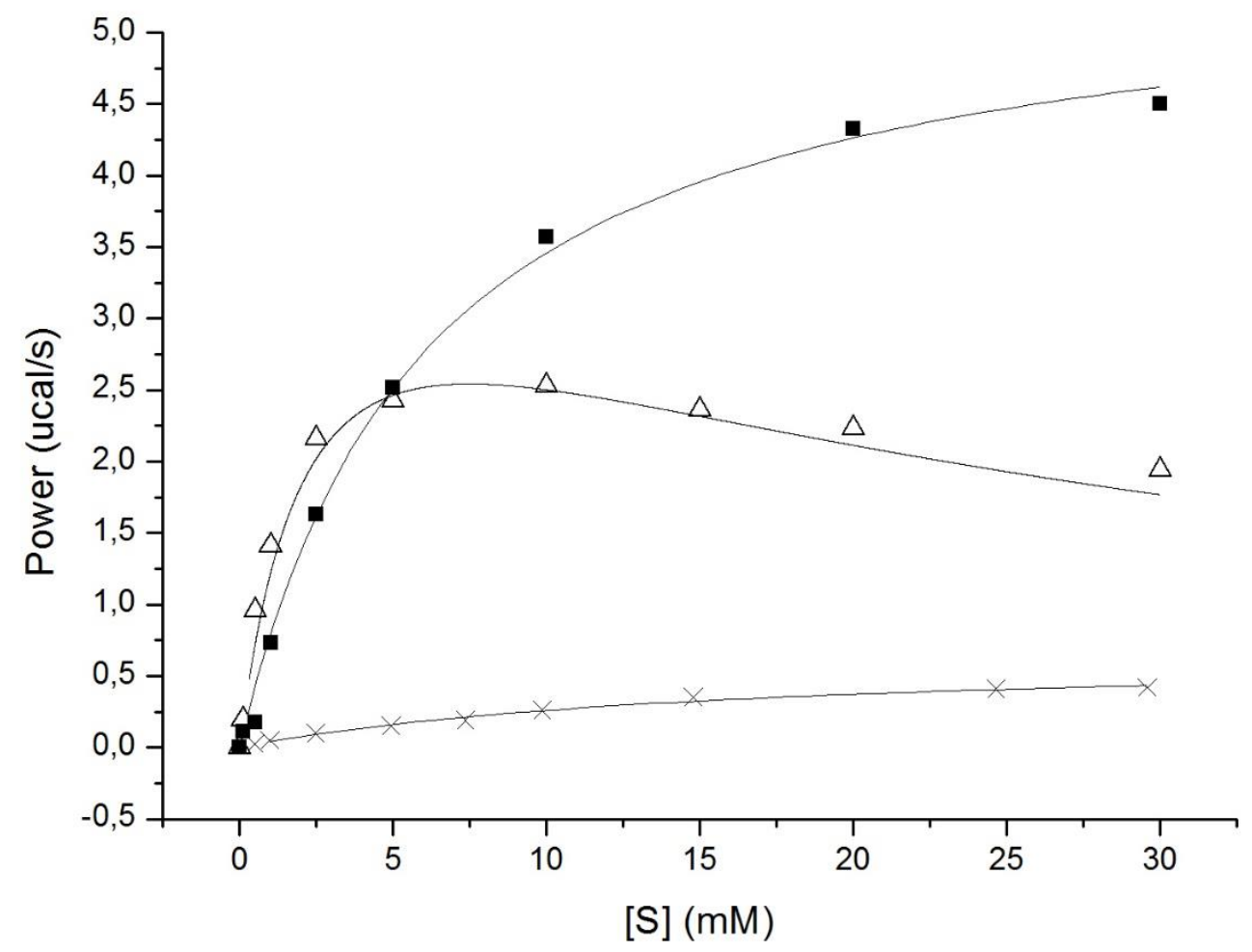

Figure 3. The effect of [G7] and [CNP-G7] on the activity of rmGPb enzyme measured by ITC. Curves were fitted with nonlinear regression applying Michaelis-Menten equation for phosphorolysis of G7 (-) as well as for synthesis reaction of CNP-G7 (x) and Haldane equation for phosphorolysis of CNP-G7 $(\Delta)$.

Substrate inhibition is often observed in nature and can play a role in metabolic feedback regulation processes in case of the natural substrate [15]. Substrate inhibition occurs when an enzyme has a second substrate binding site and two substrates can simultaneously bind to the enzyme. The experimental data for the phosphorolytic reaction of CNP-G7 (see $\Delta$ in Fig. 3) demonstrate this phenomenon; following the achievement of the maximal heat rate, an increase in 
the substrate concentration rather leads to the decrease in the reaction rate than becoming constant. It may result from the possible binding of the second substrate. Therefore, $K_{M}=2.3 \pm 0.6$ $\mathrm{mM}$ and $K_{i}=23 \pm 7 \mathrm{mM}$ were determined by using Haldane equation related to the substrate inhibition. The inhibitory effect of 4-nitrophenol (PNP) was published earlier and $I C_{50}=5 \mathrm{mM}$ was reported for the reaction of glycogen elongation in the presence of G-1-P [12]. In our study, which was carried out by ITC applying G7 and $\mathrm{P}_{\mathrm{i}}$ substrates, $I C_{50}=9 \mathrm{mM}$ was obtained for CNP in the direction of phosphorolysis. Consequently, substrate inhibition is assumed as allosteric inhibition occurring at the same binding site where the free PNP/CNP can also bind to. The remarkably high $K_{M}$ value of CNP-G7 did not allow us to experimentally achieve the saturation range of the substrate. [S] should be in the range of 0.1-10 $K_{M}$ for the precise determination of the Michaelis constant. Based on our experimental data, it is ambiguous whether substrate inhibition occurs also in the direction of synthesis, or this reaction obeys Michaelis-Menten kinetics (Fig. 3).

2.5 mM CNP-G7 was applied to the further inhibition measurements to exclude the inhibitory effect of the substrate.

\subsection{ITC activity measurement method development in both directions}

Our aim was to develop an ITC method suitable for activity measurement of GP enzyme in both directions of the catalysed reversible reaction. First, CNP-G7 was used as a model substrate for the optimization of the reaction conditions because CNP-G7 and glycogen substrates had similar $K_{M}$ values (expressed in $\mathrm{mg} / \mathrm{ml}$ ). In addition, due to the CNP chromophore group, the reaction can be monitored by HPLC. The second substrate determines the direction of the reaction: the synthesis is favoured in the presence of G-1-P, whereas the phosphorolysis reaction is favoured in the presence of inorganic phosphate. Initial substrate concentration was $2.5 \mathrm{mM}$ for each of the substrates. At the equilibrium, $58 \%$ of CNP-G7 was transformed, which resulted in longer (24 $\%$ ) and shorter (34\%) products. Varying the G-1-P/P ratio systematically, we could achieve the appropriate dominance of either the synthesis or the degradation at 10 and 0.1 ratio, respectively (upper chromatograms in Fig. 4). The same concentration ratios were applied for measurements on the glycogen substrate during the ITC inhibition studies. 


\subsection{Inhibition studies}

There are numerous known GP inhibitors [16-18], but the inhibition data of literature are not always comparable. Inhibitory parameters $\left(I C_{50}, K_{i}\right)$ of several compounds are often determined in the biologically not relevant synthesis reaction (mainly by spectrophotometry after the colour reaction of $\mathrm{P}_{\mathrm{i}}$ ) [19-22]. Breakdown reaction measurements were also carried out mainly by coupled enzyme assays [23-25]. However, measurements in both directions are less common [26].

ITC method developed in this project is suitable for activity measurements in both directions. Two well-known inhibitors were selected for our inhibition studies:

- Caffeine - as allosteric inhibitor binding to the inhibitor site - nucleotide binding site

- GTH - as glucose analogue competitive inhibitor

Prior to the ITC inhibition studies, some pre-trial were carried out on CNP-G7 substrate using HPLC technique to follow the reaction. Caffeine and GTH were applied at a concentration close to $K_{i}$. Chromatograms represented in Fig. 4 illustrate the products resulting from the reaction of breakdown and synthesis, after 5 min incubation, in the absence and presence of inhibitors.
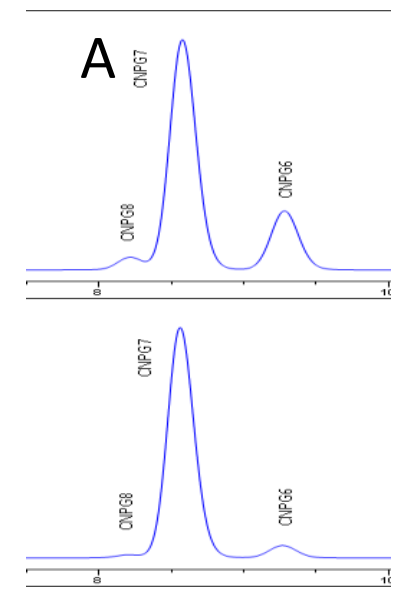
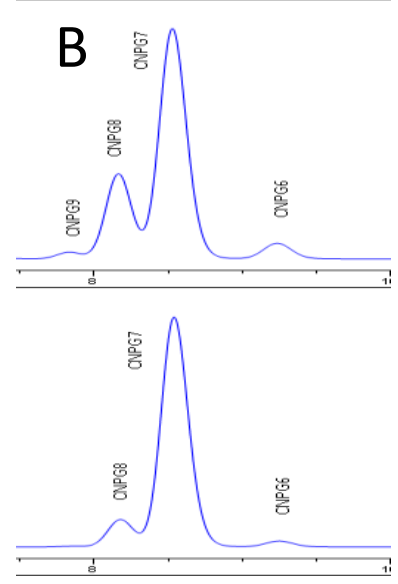
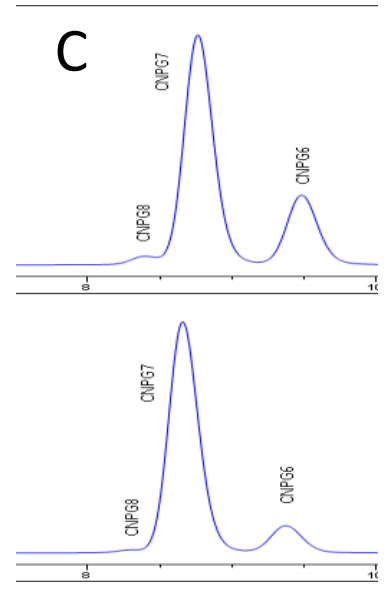
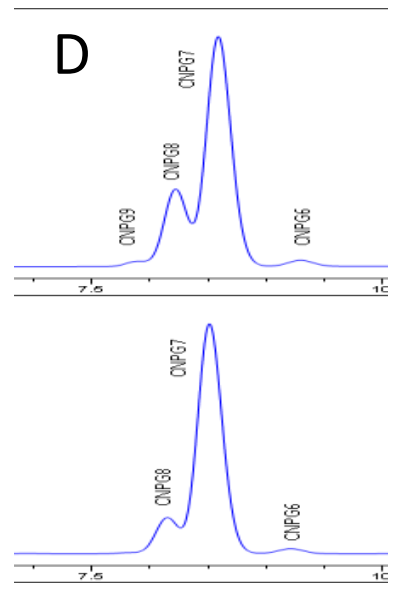

Figure 4. Phosphorolysis and synthesis of CNP-G7 substrate catalysed by rmGPb. Reaction was followed by HPLC in the absence (above) and in the presence (bottom) of inhibitors. A: GTHphosphorolysis B: GTH-synthesis C: caffeine- phosphorolysis D: caffeine- synthesis

Shorter products were produced during phosphorolysis (4A and 4C above) and longer products were produced during synthesis (4B and 4D above). The peak area of products decreased in the presence of GTH (4A and 4B bottom) and caffeine (4C and 4D bottom) inhibitors. The 
measurements were accomplished using initial rate conditions proved by the chromatograms (Fig. 4) where the main peak belongs to the CNP-G7 substrate in all cases.

ITC measurements were performed under similar reaction conditions with the exception of CNPG7, which was exchanged for glycogen substrate. Inhibitor concentration was varied and doseresponse curves were plotted. The curve fitting was accomplished with nonlinear regression, which allowed the apparent inhibition constant $\left(K_{i}^{*}\right)$ values to be determined.

\subsubsection{Study of inhibitory effect of caffeine on glycogen substrate}

Caffeine has been reported as non-competitive inhibitor affecting the purine nucleotide binding site of GPa in both the liver and the skeletal muscle. It is often applied as positive control in different inhibition studies in concentration of $1 \mathrm{mM}$ [27-30].

The results of inhibition measurements with caffeine were illustrated in Figure 5.

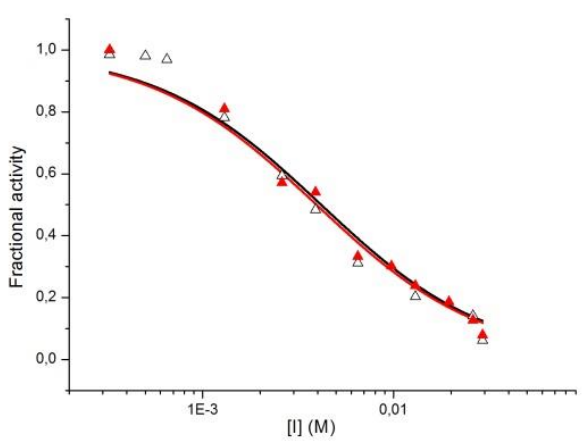

A

Figure 5. Caffeine inhibition curves of GPb enzyme catalysed reversible reaction. A) Parallel measurements of glycogen phosphorolysis. B) Parallel measurements of glycogen synthesis. Curve fitted to all points obtained from the parallel measurements in the same direction was represented by dashed line.

Model of tight binding inhibition by Greco-Hakala (Equation 5) has been fit to several sets of our experimental data. Data of parallel measurements accomplished in both reactions were in good agreement, although the match is better for the phosphorolysis reaction (Fig.5A). Apparent inhibition constants $\left(\mathrm{Ki}^{*}\right)$ provided by the curve fittings were $1.9 \pm 0.14 \mathrm{mM}$ for synthesis and 4.0 $\pm 0.26 \mathrm{mM}$ for phosphorolysis (Table 2.). Choosing total enzyme concentration as a variable 
did not change the value of $\mathrm{K}_{\mathrm{i}}{ }^{*}$, which can be replaced by $\mathrm{K}_{\mathrm{i}}$ in case of caffeine, a pure noncompetitive inhibitor. Inhibition constant of $0.1 \mathrm{mM}$ has already published for muscle and liver GPa [31], however, there are no $K_{i}$ data for $\mathrm{GPb}$ in literature.

\subsubsection{Study of inhibitory effect of GTH on glycogen substrate}

GTH (synthesized in our institute by Somsák et al.) is a very effective competitive inhibitor for $\mathrm{GPb}$ [25]. X-ray crystallographic studies revealed that this compound binds to the catalytic site of the T-state $\mathrm{GPb}$. Inhibition constants were determined in both directions (Table 2), but $K_{i}$ values proved to be different from those reported in the literature $[23,24]$.

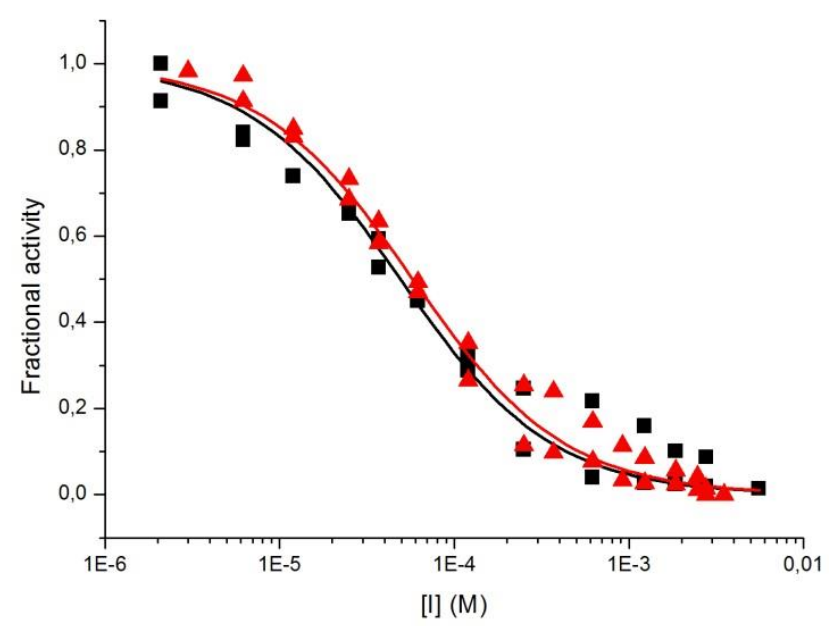

Figure 6. GTH inhibition curves of GPb enzyme catalysed reversible reactions on glycogen. Data points and fitted curves are represented by black ( $\boldsymbol{-})$ and red ( $\boldsymbol{\Delta})$ for synthesis and phosphorolysis reaction, respectively.

The inhibition curves fitted to our experimental data (Fig. 6) are very similar and $K i^{*}$ values are also close to each other; $48.9 \pm 4.1 \mu \mathrm{M}$ and $59.9 \pm 3.2 \mu \mathrm{M}$ for the directions of synthesis and phosphorolysis, respectively.

$K_{i}$ values of $11.3 \mu \mathrm{M}$ and $13,8 \mu \mathrm{M}$ were calculated from $K i^{*}$ using Equation (5), where $[S]=5$ $\mathrm{mg} / \mathrm{ml}$ and $K_{M}=1.5 \mathrm{mg} / \mathrm{ml}$ were applied for glycogen (Table 2). 


\section{Conclusion}

ITC, in addition to its prevalent role in binding studies, is a promising method in enzymatic assays due to the elimination of the need of synthetic chromophores, fluorophores, radioactive labels on substrate or of indirect coupled assays. A further advantage of the ITC method is that the obtained data are directly proportional to the reaction rate, in contrast with the discontinuous measurements where the concentration-time relationship must be determined. Classical ITC methods (single or multiple injections) for enzyme kinetic measurements [10] are not suitable for the study of reversible enzyme reactions since the products are also the substrates for the reverse reaction. Instead of complete substrate depletion, necessary for a single injection ITC method, an equilibrium mixture is formed where the rates of forward and reverse reactions are equal. This may be one of the reasons why ITC method has been mainly used for studying hydrolases where the reverse reaction is negligible due to the presence of a large amount of water [32-36]. Using $\mathrm{rmGPb}$ as a model for sugar phosphorylases we have established the conditions suitable for activity measurements in both directions of the reversible reaction.

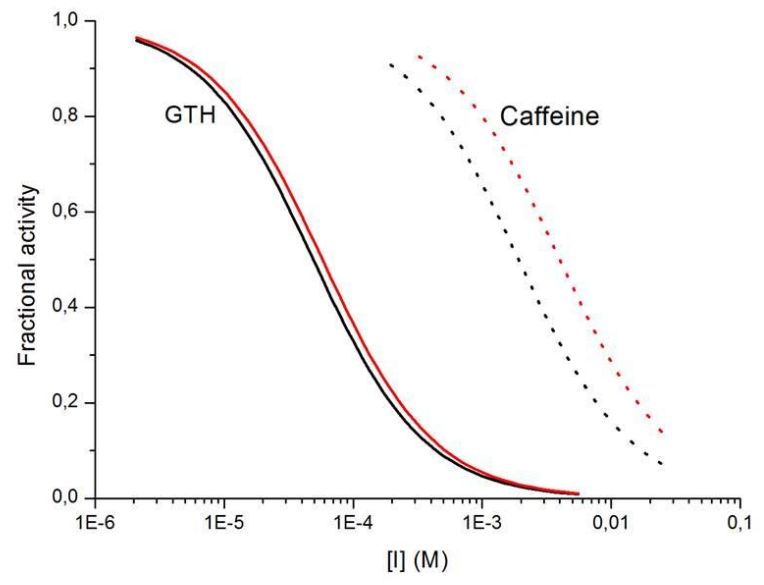

Figure 7. Summary of inhibition measurements on the GPb-glycogen reaction in the presence of GTH and caffeine. Curves were fitted on all the points obtained from the parallel measurements carried out in the direction of synthesis (black) and phosphorolysis (red).

Inhibition measurements demonstrated that inhibiton constants are almost the same in case of competitive inhibitor, while the difference is higher for non-competitive inhibitor. In competitive inhibition enzyme-inhibitor complex (EI) is formed, which can be characterized by a well- 
defined, single inhibition constant $\left(\mathrm{K}_{\mathrm{EI}}\right)$. $\mathrm{K}_{\mathrm{EI}}$ must be the same in both forward and reverse reactions. However, in case of non-competitive inhibition two types of inhibitor complex are formed EI and ESI. Therefore, two inhibition constants ( $\mathrm{K}_{\mathrm{EI}}$ and $\left.\mathrm{K}_{\mathrm{ESI}}\right)$ are necessary for the description of inhibition. In the reversible reaction catalysed by glycogen phosphorylase the substrates and their concentrations are different in synthetic and phosphorolytic reaction resulted in different $\mathrm{K}_{\mathrm{ESI}}$ values. Even if all microscopic dissociation constants are identical, they differ from the macroscopic ones, and calculated $\mathrm{K}_{\mathrm{i}}$ values can be different for synthesis and phosphorolysis.

The results presented here have demonstrated that the ITC method, using the developed experimental design and reaction conditions, is suitable for activity measurements of different phosphorylase enzymes on various substrates in both directions and is applicable for inhibition studies as well. Although under physiological conditions the glycogen breakdown is favoured by $\mathrm{rmGPb}$, there are some other phosphorylases preferring synthesis rather than phosphorolysis reaction [37]. Our new method can be very useful for the examination of similar reversible processes in order to determine the preferences of the enzymes.

\section{Experimental}

\section{$\underline{4.1 . ~ M a t e r i a l s ~}$}

Glycogen phosphorylase $b$ enzyme from rabbit muscle, AMP, EDTA and free maltooligosaccharide substrates (maltopentaose: G5, maltoheptaose: G7), were purchased from SIGMA Aldrich (St. Louis, MO, USA). Oyster glycogen $\left(\overline{M w}=85000 \mathrm{gmol}^{-1}\right)$ was obtained from Merck (Darmstadt, Germany). Glucose-1-phosphoric acid dipotassium salt 14ehydrate was purchased from Reanal-Across (Budapest, Hungary). Caffeine was acquired from WHO International Chemical Reference Substances (Stockholm, Sweden). 2-Chloro-4-nitrophenyl- $\beta$ maltoheptaoside (CNP-G7) and GTH were synthesized at the University of Debrecen [38, 39].

\subsection{Enzyme assays}

For the substrate specificity measurements $1 \mathrm{M}$ phosphate buffer containing $2.6 \mathrm{mM}$ EDTA and 4 mM AMP was applied in the direction of phosphorolysis, whereas $35.4 \mathrm{mM} \beta$-glycerophosphate 
buffer containing $2.6 \mathrm{mM}$ EDTA, $4 \mathrm{mM}$ AMP, 50mM G-1-P was used in the direction of synthesis.

During the inhibition studies each reactant (enzyme, glycogen or CNP-G7 and inhibitor) was dissolved in $35 \mathrm{mM}$ glycerophosphate buffer ( $\mathrm{pH}$ 6.8) containing 2.6 mM EDTA, $1 \mathrm{mM}$ AMP 2.5 $\mathrm{mM} \mathrm{P} \mathrm{P}_{\mathrm{i}}$ and $25 \mathrm{mM} \mathrm{G}-1-\mathrm{P}$ for synthesis or $25 \mathrm{mM} \mathrm{P} 2.5 \mathrm{mM} \mathrm{G}-1-\mathrm{P}$ for breakdown. Enzyme reactions were carried out at $37^{\circ} \mathrm{C}$.

\subsubsection{Isothermal titration calorimetry}

ITC experiments were carried out with an $\mathrm{ITC}_{200}$ microcalorimeter (MicroCal, Northampton, MA, USA) at $37^{\circ} \mathrm{C}$ using similar experimental layout to our recently published method [40]. In each measurement rmGP solution was injected $(5 \mu \mathrm{L} / 10 \mathrm{~s})$ into the sample cell containing the substrate solution $(5 \mathrm{mg} / \mathrm{ml}$ glycogen, in synthesis or breakdown buffer) in the presence or absence of inhibitors. In each experiment heat flow $(d Q / d t)$ was measured in "low-gain" feedback mode until its absolute value reached the maximum at each different substrate or inhibitor concentrations. Origin 7 software (Northampton, MA, USA) was applied for evaluating the experimental raw ITC data.

In addition, $\Delta H$ was determined for phosphorolysis using the usual single injection method. The reaction was initiated by injecting GPb enzyme solution into the sample cell containing $15 \mathrm{mM}$ G5 substrate dissolved in $1 \mathrm{M}$ phosphate buffer. The reaction could go to completion in the calorimeter cell, and the power $P$ was recorded as a function of time $t$. Integration of the excess power $P$ associated with the reaction enables $\Delta H$ to be determined according to Equation (2) [41].

$$
\Delta \mathrm{H}=\frac{\int_{0}^{\infty} \mathrm{Pdt}}{[\mathrm{S}]_{\mathrm{t}=0} \mathrm{~V}^{0}}
$$

This calculated value of $\Delta H$ was applied only for the determination of $k_{c a t} / K_{m}$ (see in Table 1.).

\subsubsection{HPLC method for GP b activity measurement}

The enzyme solution $(4 \mathrm{mg} / \mathrm{ml})$ was prepared in buffer $\mathrm{pH}$ 6.8. For each measurement $2.5 \mathrm{mM}$ CNP-G7 and $2.5 \mathrm{mM}-25 \mathrm{mM} \mathrm{G}-1-\mathrm{P}$ or $\mathrm{P}_{\mathrm{i}}$ substrates were freshly dissolved in buffer. The mixture of $14 \mu \mathrm{l}$ enzyme and $1 \mathrm{ml}$ substrate was homogenized with vortex and incubated at $37^{\circ} \mathrm{C}$ for 5 minutes. $5 \mu \mathrm{l}$ of the solution was injected into the column in every 15 minutes, 4 times in total. Agilent 1260 Infinity 2 instrument equipped with quaternary pump, degasser and automata sample injector was used for the measurements. Separation was made using a Hypersil ODS (20 
$\mathrm{cm} \times 4.6 \mathrm{~mm} 120 \AA 5 \mu \mathrm{m}$, Agilent) column. Isocratic elution was applied with $\mathrm{MeCN} / \mathrm{H}_{2} \mathrm{O}$ $(12: 88, \mathrm{v} / \mathrm{v} \%)$ and with the flow rate of $1 \mathrm{ml} / \mathrm{min}$. The column temperature was set to $30^{\circ} \mathrm{C}$. Detection of CNP-G7 and longer or shorter CNP-oligomer products were carried out at 302nm. Summarized area data of CNP-oligomer products were plotted in the function of reaction time to determine the reaction rate. Linear regression was used and the slope of the fitted linear curve provided the enzyme activity.

\subsection{Evaluation of kinetic data}

Heat flow values, considered as reaction rate data, originated from ITC measurements were evaluated in the same way for each experiment. All plotting and fitting procedures were accomplished by using the Origin software applying the adequate equations. In order to determine the $K_{M}$ and $K_{i}$ values, heat flow $(d Q / d t)$ data proportional to $\mathrm{v}_{0}$ were plotted as a function of substrate concentrations and a curve was fitted with nonlinear regression based on MichaelisMenten and Haldane equations [42] (Equation (3) and (4), respectively),

$$
\begin{gathered}
v=\frac{v_{\max } \times[S]}{K_{M}+[S]} \\
v=\frac{v_{\max } \times[S]}{K_{M}+[S]+\frac{[S]^{2}}{K_{i}}}
\end{gathered}
$$

where $v$ is the reaction rate, $v_{\max }$ is the maximal reaction rate at saturating substrate concentration, [S] is the substrate concentration, $K_{M}$ is the Michaelis constant and $K_{i}$ is the inhibition constant.

The experimental data of inhibition measurements were evaluated applying Greco-Hakala relative rate equation [43] below.

$$
\frac{\mathrm{v}}{\mathrm{v}_{0}}=\frac{\mathrm{E}_{\mathrm{t}}-\mathrm{I}-\mathrm{K}_{\mathrm{i}}^{*}+\sqrt{\left(\mathrm{E}_{\mathrm{t}}-\mathrm{I}-\mathrm{K}_{\mathrm{i}}^{*}\right)^{2}+4 \mathrm{E}_{\mathrm{t}} * \mathrm{~K}_{\mathrm{i}}^{*}}}{2 \mathrm{E}_{\mathrm{t}}}
$$

$\mathrm{Ki}^{*}=\mathrm{Ki}(1+[\mathrm{S}] / \mathrm{Km})$ for competitive inhibition

$\mathrm{Ki}^{*}=\mathrm{Ki}$ for non competitive inhibition

where the dependent variable is the relative reaction rate $(v / v o), K_{i} *$ is the apparent inhibition constant, $E_{t}$ is the total enzyme concentration, $I$ is the inhibitor concentration (independent variable), $[S]$ is the substrate concentration and $K_{m}$ is the Michaelis constant.

The equation was inserted to the Origin as user defined relationship. 


\section{Acknowledgements}

The research was supported by the EU and co-financed by the European Regional Development Fund under the project GINOP-2.3.2-15-2016-00008. The authors are grateful for the financial support of the ITC equipment by TÁMOP-4.2.1.-15-B-09/1/KONV and OTKA CK77515. We also thank Csaba Hámori PhD student for carrying out the HPLC measurements.

\section{References}

[1] S. Tanabe, M. Kobayashi, K. Matsuda, Yeast glycogen phosphorylase: kinetic properties compared with muscle and potato enzymes, Agricultural Biological Chemistry 52 (1988) 757764. https://doi.org/10.1080/00021369.1988.10868723.

[2] W.E. Schreiber, S. Bowling, An automated assay of glycogen phosphorylase in the direction of phosphorolysis, Ann. Clin. Biochem. 27 (1990) 129-132. DOI: 10.1177/000456329002700207.

[3] C.J. Zea, N.L. Pohl, Kinetic and substrate binding analysis of phosphorylase b via electrospray ionization mass spectrometry: A model for chemical proteomics of sugar phosphorylases, Analytical Biochemistry 327 (2004) 107-113. DOI: 10.1016/j.ab.2003.12.022.

[4] N. Alonso-Casajús, D. Dauvillée, A.M. Viale, F.J. Muñoz, E. Baroja-Fernández, M.T. MoránZorzano, G. Eydallin, S. Ball , J. Pozueta-Romero, Glycogen Phosphorylase, the Product of the $\operatorname{glg} P$ Gene, Catalyzes Glycogen Breakdown by Removing Glucose Units from the Nonreducing Ends in Escherichia coli, Journal of Bacteriology 188 (2006) 5266-5272. DOI: 10.1128/JB.01566-05.

[5] M. Nakamura, Y. Makino, C. Takagi, T. Yamagaki, M. Sato, Probing the catalytic site of rabbit muscle glycogen phosphorylase using a series of specifically modified maltohexaose derivatives, Glycoconjugate Journal 34 (2017) 563-574. DOI: 10.1007/s10719-017-9776-5.

[6] C.H. Fiske, Y. Subbarow, The colorimetic determination of phosphorus, The Journal of Biological Chemistry 66 (1925) 375-400.

[7] D.P. Gilboe, K.L. Larson, F.Q. Nuttall, Radioactive Method for the Assay of Glycogen Phosphorylases, Analytical Biochemistry 47 (1972) 20-27. https://doi.org/10.1016/00032697(72)90274-6.

[8] Y. Makino, K. Omichi, Sensitive assay of glycogen phosphorylase activity by analysing the chainlengthening action on a fluorogenic maltooligosaccharide derivative, Journal of Biochemistry 146 (2009) 71-76. DOI: 10.1093/jb/mvp044.

[9] P.L. Mateo, C. Barón, O. Lopez-Mayorga, J.S. Jimenez, M. Cortijo, AMP and IMP binding to glycogen phosphorylase b. A calorimetric and equilibrium dialysis study, Journal of Biological Chemistry 259 (1984) 9384-9389.

[10] M.J. Todd, J. Gomez, Enzyme Kinetics Determined Using Calorimetry: A General Assay for Enzyme Activity?, Analytical Biochemistry 296 (2001) 179-187. DOI: 10.1006/abio.2001.5218. 
[11] S.A. Assaf, A.A. Yunis, Physicochemical and catalytic properties of crystallized human muscle glycogen phosphorylase, Annals of The New York Academy of Science 210 (1973) 139-152.

[12] M. Ariki, T. Fukui, Inhibition of alpha-glucan phosphorylase by alpha-D-glucopyranosyl fluoride, J. Biochem. 78 (1975) 1191-1199.

[13] O. Anderka, P. Loenze, T. Klabunde, M.K. Dreyer, E. Defossa, K.U. Wendt, D. Schmoll, Thermodynamic characterization of allosteric glycogen phosphorylase inhibitors, Biochemistry 47 (2008) 4683-4691. DOI: 10.1021/bi702397d.

[14] S. Cecioni, O.A. Argintaru, T. Docsa, P. Gergely, J.P. Praly, S. Vidal, Probing multivalency for the inhibition of an enzyme: glycogen phosphorylase as a case study, New J. Chem. 33 (2009) 148-156. https://doi.org/10.1039/b812540f.

[15] M.C. Reed, A. Lieb, H.F. Nijhout, The biological significance of substrate inhibition: a mechanism with diverse functions, Bioessays 32 (2010) 422-429. DOI: 10.1002/bies.200900167.

[16] L. Somsák, K. Czifrák, M. Tóth, É. Bokor, E.D. Chrysina, K.M. Alexacou, J.M. Hayes, C. Tiraidis, E. Lazoura, D.D. Leonidas, S.E. Zographos, N.G. Oikonomakos, New Inhibitors of Glycogen Phosphorylase as Potential Antidiabetic Agents, Current Medicinal Chemistry 15 (2008) 2933-2983. https://doi.org/10.2174/092986708786848659.

[17] K.M. Alexacou, A.C. Tenchiu Deleanu, E.D. Chrysina, M.D. Charavgi, I.D. Kostas, S.E. Zographos, N.G. Oikonomakos, D.D. Leonidas, The binding of beta-D-glucopyranosylthiosemicarbazone derivatives to glycogen phosphorylase: a new class of inhibitors, Bioorg. Med. Chem. 18 (2010) 7911-7922. DOI: 10.1016/j.bmc.2010.09.039.

[18] A. Kato, N. Nasu, K. Takebayashi, I. Adachi, Y. Minami, F. Sanae, N. Asano, A.A. Watson, R.J. Nash, Structure-activity relationships of flavonoids as potential inhibitors of glycogen phosphorylase, J. Agric. Food Chem. 56 (2008) 4469-4473. DOI: 10.1021/jf800569s.

[19] N.G. Oikonomakos, S.E. Zographos, V.T. Skamnaki, G. Archontis, The 1.76 A resolution crystal structure of glycogen phosphorylase B complexed with glucose, and CP320626, a potential antidiabetic drug, Bioorg. Med. Chem. 10 (2002) 1313-1319.

[20] N.G. Oikonomakos, J.B. Schnier, S.E. Zographos, V.T. Skamnaki, K.E. Tsitsanou, L.N. Johnson, Flavopiridol inhibits glycogen phosphorylase by binding at the inhibitor site, J. Biol. Chem. 275 (2000) 34566-73. DOI: 10.1074/jbc.M004485200.

[21] W.H. Martin, D.J. Hoover, S.J. Armento, I.A. Stock, R.K. McPherson, D.E. Danley, R.W. Stevenson, E.J. Barrett, J.L. Treadway, Discovery of a human liver glycogen phosphorylase inhibitor that lowers blood glucose in vivo, Proc. Natl. Acad. Sci. USA 95 (1998) 1776-1781.

[22] R. Mosi, S.G. Withers, Synthesis and kinetic evaluation of 4-deoxymaltopentaose and 4deoxymaltohexaose as inhibitors of muscle and potato alpha-glucan phosphorylases, Biochem. J. 338 (1999) 251-256.

[23] N.G. Oikonomakos, V.T. Skamnaki, E. Ösz, L. Szilágyi, L. Somsák, T. Docsa, B. Tóth, P. Gergely, Kinetic and Crystallographic Studies of Glucopyranosylidene Spirothiohydantoin Binding to Glycogen Phosphorylase b, Bioorganic \& Medicinal Chemistry 10 (2002) 261-268. https://doi.org/10.1016/S0968-0896(01)00277-2.

[24] L. Somsák, L. Kovács, M. Tóth, E. Osz, L. Szilágyi, Z. Györgydeák, Z. Dinya, T. Docsa, B. Tóth, P. Gergely, Synthesis of and a comparative study on the inhibition of muscle and liver glycogen phosphorylases by epimeric pairs of d-gluco- and d-xylopyranosylidene-spiro(thio)hydantoins and N-(d-glucopyranosyl) amides, J Med Chem. 44 (2001) 2843-2848.

[25] E.P. Mitchell, S.G. Withers, P. Ermert, A.T. Vasella, E.F. Garman, N.G. Oikonomakos, L.N. Johnson, Ternary Complex Crystal Structures of Glycogen Phosphorylase with the Transition 
State Analogue Nojirimycin Tetrazole and Phosphate in the T and R States, Biochemistry 35 (1996) 7341-7355. DOI: 10.1021/bi960072w.

[26] L. Somsak, V. Nagy, Z. Hadady, T. Docsa, P. Gergely, Glucose Analog Inhibitors of Glycogen Phosphorylases as Potential Antidiabetic Agents: Recent Developments, Current Pharmaceutical Design 9 (2003) 1177-1189.

[27] S. Freeman, J.B. Bartlett, G. Convey, J.L. Hardern, J.L. Teague, S.J.G. Loxham, J.M. Allen, S.M. Poucher, A.D. Charles, Sensitivity of glycogen phosphorylase isoforms to indole site inhibitors is markedly dependent on the activation state of the enzyme, Br J Pharmacol. 149 (2006) 775-785. DOI: 10.1038/sj.bjp.0706925.

[28] V.L. Rath, M. Ammirati, D.E. Danley, J.L. Ekstrom, E.M. Gibbs, T.R. Hynes, A.M. Mathiowetz, R.K. McPherson, T.V. Olson, J.L. Treadway, D.J. Hoover, Human liver glycogen phosphorylase inhibitors bind at a new allosteric site, Chemistry \& Biology 7 (2000) 677-682.

[29] K. Cheng, P. Zhang, J. Liu, J. Xie, H. Sun, Practical synthesis of bredemolic acid, a natural inhibitor of glycogen phosphorylase, J Nat Prod. 71 (2008) 1877-1880. DOI: $10.1021 / n p 8003886$.

[30] P.J. Kasvinsky, S. Shechosky, R.J. Fletterick, Synergistic Regulation of Phosphorylase a by Glucose and Caffeine, The Journal of Biological Chemistry 253 (1978) 9102-9106.

[31] K. Cheng, J. Liu, H. Sun, J. Xie, Synthesis of glucoconjugates of oleanolic acid as inhibitors of glycogen phosphorylase, Chemistry \& Biodiversity 7 (2010) 690-696. DOI: 10.1016/j.carres.2009.02.012.

[32] N. Karim, H. Okada, S. Kidokoro, Calorimetric evaluation of the activity and the mechanism of cellulases for the hydrolysis of cello- oligosaccharides accompanied by the mutarotation reaction of the hydrolyzed products, Thermochim Acta 412 (2004) 91-6. DOI: 10.1016/j.tca.2005.01.025.

[33] T. Jeoh, J.O. Baker, M.K. Ali, M.E. Himmel, W.S. Adney, Beta-D-Glucosidase reaction kinetics from isothermal titration microcalorimetry, Anal Biochem 347 (2005) 244-53. DOI: 10.1016/j.ab.2005.09.031.

[34] M.J. Baumann, L. Murphy, N. Lei, K.B.R.M. Krogh, K. Borch, P. Westh, Advantages of isothermal titration calorimetry for xylanase kinetics in comparison to chemical-reducing-end assays, Anal Biochem 410 (2011) 19-26. DOI: 10.1016/j.ab.2010.11.001.

[35] P. Draczkowski, A. Tomaszuk, P. Halczuk, M. Strzemski, D. Matosiuk, K. Jozwiak, Determination of affinity and efficacy of acetylcholinesterase inhibitors using isothermal titration calorimetry, BBA Gen Subjects 1860 (2015) 967-974. DOI: 10.1016/j.bbagen.2015.11.006.

[36] K. Maximova, J. Trylska, Kinetics of trypsin catalyzed hydrolysis determined by isothermal titration calorimetry, Anal Biochem 486 (2015) 24-34. DOI: 10.1016/j.ab.2015.06.027.

[37] J. Jiang, C. Yao, X. Cao, Y. Liu, S. Xue, Characterization of starch phosphorylase from the marine green microalga (Chlorophyta) Tetraselmis subcordiformis reveals its potential role in starch biosynthesis, J. Plant Physiol. 218 (2017) 84-93. DOI: 10.1016/j.jplph.2017.07.019.

[38] E. Farkas, L. Jánossy, J. Harangi, A. Lipták, Synthesis of chromogenic substrates of $\alpha$ amylases on a cyclodextrin basis, Carbohydrate Research 303 (1997) 407-415. https://doi.org/10.1016/S0008-6215(97)00187-0

[39] L. Somsak, V. Nagy, Z. Hadady, T. Docsa, P. Gergely, Glucose analog inhibitors of glycogen phosphorylases as potential antidiabetic agents: recent developments, Curr. Pharm. Des. 9 (2003) 1177-1189. 
[40] G. Lehoczki, K. Szabó, I. Takács, L. Kandra, G. Gyémánt, Simple ITC method for activity and inhibition studies on human salivary $\alpha$-amylase, Journal of Enzyme Inhibition and Medicinal Chemistry 31 (2016) 1648-1653. DOI: 10.3109/14756366.2016.1161619.

[41] MicroCal. ITC Data Analysis in Origin ${ }^{\circledR}$ Tutorial Guide. MicroCal, LLC; Northampton, MA: 2004.

[42] J. R. Sonnad, C. T. Goudar, Solution of the Haldane Equation for Substrate Inhibition Enzyme Kinetics Using the Decomposition Method, Mathematical and Computer Modelling 40 (2004) 573-582. https://doi.org/10.1016/j.mcm.2003.10.051.

[43] Kuzmiĉ, P. (2015) History, variants and usage of the "Morrison equation" in enzyme inhibition kinetics, BioKin Technical Note TN-2015-01, BioKin Ltd., Watertown MA, [Online]www.biokin.com/TN/2015/01 